\title{
Composición química y producción de follaje de Erythrina americana (Fabaceae) en cercos vivos durante dos épocas climáticas
}

\section{Jorge Oliva-Hernández ${ }^{1}$, María Aurelia López-Herrera ${ }^{2}$ \& Erika Belem Castillo-Linares ${ }^{1}$}

1. Instituto Nacional de Investigaciones Forestales, Agrícolas y Pecuarias, Campo Experimental Huimanguillo, km 1 carretera Huimanguillo-Cárdenas, 86400. Huimanguillo, Tabasco, México; oliva.jorge@inifap.gob.mx, castillo.erika@inifap.gob.mx

2. Instituto Nacional de Investigaciones Forestales, Agrícolas y Pecuarias, Campo Experimental Mocochá, Calle 6 número 398 x 13, Avenida Correa Rachó, 97130. Yucatán, México; lopez.aurelia@inifap.gob.mx

Recibido 15-V-2020. Corregido 14-IX-2020. Aceptado 21-X-2020.

\begin{abstract}
Chemical composition and foliage production of Erythrina americana (Fabaceae) in living fences during two climatic seasons. Introduction: Erythrina americana is a tree legume found as part of the fence of livestock farms of the tropical region. However, sheep farmers do not routinely or strategically use the foliage of this type of legume as a dietary supplement due, in part, to ignorance of its chemical composition and foliar performance, as well as the factors that affect them. Objective: To determine the influence of season and diameter of E. americana tree on the chemical composition and foliage yield at a 90-day regrowth age. Methods: 47 trees were used in a two-factor design. The factors were season (dry and rainy) and tree diameter at $0.20 \mathrm{~m}$ above ground level (D-9 between 0.05 and $0.09 \mathrm{~m}$ and D-13, between 0.091 to $0.13 \mathrm{~m}$ ). The response variables were dry matter production and crude protein from foliage ( $\mathrm{kg}^{2}$ rbol $\left.{ }^{-1}\right)$. Results: The season did not affect $(\mathrm{P}>0.05)$ the production of dry matter and crude protein. However, the diameter of E. americana affected them $(\mathrm{P}<0.01)$. In diameter $\mathrm{D}-13$, the highest production of dry matter and crude foliar protein tree $^{-1}$ was detected with respect to D-9. Conclusions: The diameter of the tree had a more influence than the season on yield of dry matter and crude protein.
\end{abstract}

Key words: humid tropic; live fence; sheep feeding; tree legume.

Oliva-Hernández, J., López-Herrera, M.A., \& Castillo-Linares, E.B. (2021). Composición química y producción de follaje de Erythrina americana (Fabaceae) en cercos vivos durante dos épocas climáticas. Revista de Biología Tropical, 69(1), 90-101. DOI 10.15517/rbt.v69i1.41822

Erythrina americana es una leguminosa arbórea que es considerada como árbol multiusos (García-Mateos, Soto-Hernández, \& Vibrans, 2001; Villanueva, Martínez, \& Van der Wal, 2015). Se ha propuesto su uso para la restauración de bosques, en especial de aquellos localizados en la región tropical con estación seca (Fehling-Fraser \& Ceccon, 2015). En México, se ha documentado el uso etnomédico de sus flores y semillas (García-Mateos et al., 2001; Pino, Prieto, Pérez, \& Molina, 2004; Sotelo, López-García, \& Basurto-Peña,
2007). En ganadería se emplea como parte del cerco (poste vivo), proporcionando de manera adicional sombra y leña, así como, ejerciendo la función de cortina rompevientos (Llera \& Meléndez, 1994; Grande, Villanueva, Maldonado, \& Hernández, 2013; Oliva-Hernández, López-Herrera, Velázquez-Jiménez, LópezEnríquez, \& Vélez-Pérez, 2019). En el estado de Tabasco, en los cercos vivos de fincas ganaderas se han registrado al menos 100 especies arbóreas, encontrándose entre dos y hasta 12 especies distintas en un mismo cerco. En dos 
de los 17 municipios de Tabasco: Cárdenas y Huimanguillo, E. americana se identifica como una de las tres especies con mayor presencia en los cercos vivos (Grande et al., 2013).

En cuanto a la agricultura desarrollada en Tabasco, E. americana se utiliza principalmente para proporcionar sombra en las plantaciones de cacao (Theobroma cacao L.). Adicionalmente, esta especie produce otros beneficios: ayuda a conservar y mejorar el suelo y a propiciar al aumento de rendimiento de semilla de cacao (Sol-Sánchez, López-Juárez, CórdovaÁvalos, \& Gallardo-López, 2018). Además, Erythrina sp. se puede encontrar como parte de los huertos familiares localizados en las regiones rurales de Tabasco (Chablé-Pascual et al., 2015), en donde las hojas de esta especie se emplean con fines medicinales y sus inflorescencias como alimento para humanos (Centurión, Cázares, Espinosa, Poot Matu, \& Mijangos, 2003; Sotelo et al., 2007; GarcíaFlores, González-Espinosa, Lindig-Cisneros, \& Casas, 2019). Sin embargo, cabe señalar que la leña que produce esta especie es de baja calidad maderable (Sol-Sánchez et al., 2018).

Aunque Erythrina forma parte de los recursos forestales en fincas ovinas localizadas en la región tropical, los productores de ovinos no utilizan este tipo de follaje como complemento alimenticio de forma rutinaria o estratégica, debido principalmente a que desconocen su composición química y la cantidad que se puede ofrecer a los ovinos (Maldonado et al., 2008; Candelaria-Martínez, Flota-Bañuelos, \& Castillo-Sánchez, 2015; Morantes-Toloza \& Renjifo, 2018). Tomando en cuenta que el follaje de Erythrina tiene un contenido de proteína bruta (PB) de entre 14.5 y $25.6 \%$, y de fibra detergente neutro (FDN) entre 52.4 y 71.6 $\%$, se puede hacer factible su uso como complemento alimenticio para pequeños rumiantes (Oliva-Hernández et al., 2019; HernándezEspinoza et al., 2020b). Además, los ovinos consumen con facilidad este tipo de follaje y se puede incorporar en su dieta hasta en un 30 $\%$ e incluso como único alimento por períodos cortos, de hasta 28 días (Best et al., 2017; Hernández-Espinoza et al., 2020b). Erythrina puede producir follaje todo el año, cuando es sometida a un proceso de poda controlada y se han determinado las edades de rebrote de 90 y 120 días como las de mayor producción de MS con relación a 30 y 60 días (Meléndez, 2003). De acuerdo con Hernández-Espinoza et al. (2020a) a los 90 días de rebrote la PB del follaje es mayor $(17.3 \%)$ con respecto a $60 \mathrm{y}$ 120 días, con 15.7 y $15.4 \%$, respectivamente.

Sin embargo, para hacer un uso intensivo de E. americana como proveedor de follaje se requiere conocer la influencia de diversos factores sobre la producción del mismo, así establecer prácticas de manejo en los árboles para optimizar su calidad y predecir su producción. Por ejemplo, en Gliricidia sepium la altura de poda y el ecotipo son factores que influyen en el rendimiento de biomasa (Gómez, Molina, Molina, \& Murgueitio, 1990; Ramos-Trejo, Canul-Solis, \& Ku-Vera, 2016). Para el caso de Erythrina, la información sobre la influencia de la época del año sobre la composición química y producción del follaje es escasa, sobre todo para las condiciones del trópico en las que las diferencias en precipitación pluvial y temperatura ambiente a través del año pueden afectar ambas características. Además, otros factores como el diámetro y la edad de rebrote deben considerarse debido a que puede influir la producción de follaje, y ser de utilidad para proyectar la producción y disponibilidad de follaje en un cerco vivo. El objetivo de este estudio consistió en determinar la influencia de la época climática y diámetro del árbol $E$. americana en la composición química y rendimiento de follaje a una edad de rebrote de 90 días.

\section{MATERIALES Y MÉTODOS}

Localización: El estudio se realizó en los cercos vivos de la Unidad Experimental Ovina del Instituto Nacional de Investigaciones Forestales, Agrícolas y Pecuarias (INIFAP), localizada en el municipio de Huimanguillo, Tabasco, México (17 $51^{\prime} 4^{\prime \prime} \mathrm{N} \& 93^{\circ} 23^{\prime} 47^{\prime}$ ' W). El clima es cálido húmedo, con lluvias todo el año (Af), temperatura ambiente media anual de $26.2^{\circ} \mathrm{C}$, oscilación térmica $10.6^{\circ} \mathrm{C}$, 
precipitación pluvial media anual $2386.0 \mathrm{~mm}$, evaporación $954.1 \mathrm{~mm}$ y fotoperiodo $12 \mathrm{~h}$ (Díaz, Ruíz, Medina, Cano, \& Serrano, 2006; INEGI, 2017).

Condiciones climáticas: Se consultaron los registros climáticos de la estación meteorológica de la Comisión Nacional del Agua (CONAGUA, 2017) localizada en el Campo Experimental Huimanguillo, INIFAP. Con los datos climáticos se determinó el promedio en la temperatura ambiente (mínima, media y máxima, ${ }^{\circ} \mathrm{C}$ ), humedad relativa (mínima, media y máxima, \%), precipitación pluvial acumulada $(\mathrm{mm})$ y radiación solar $\left(\mathrm{Wm}^{-2}\right)$ desde la fecha de corte de uniformidad hasta la duración de la edad de rebrote. El estudio se desarrolló durante dos épocas climáticas subsecuentes: transición de nortes a sequía, enero a abril de 2017 (sequía) y transición de sequía a lluvias, mayo a julio de 2017 (lluvias).

Árboles, poda y follaje: Los árboles utilizados en el estudio formaron parte de un grupo de 140 árboles localizados en el cerco perimetral de las praderas manejadas para el pastoreo de ovinos (sembrados como varetas entre 2009 y 2012). Estos árboles tuvieron un manejo de poda controlada a intervalos de 90 días, de forma que previo al estudio, éstos habían recibido tres podas de todas las ramas. A partir del total de árboles, se seleccionaron los que estuvieran rectos y sin ramificaciones; con una distancia entre árboles cercana a $1 \mathrm{~m}$; y con un diámetro a $0.20 \mathrm{~m}$ del suelo mayor a $0.05 \mathrm{~m}$ y menor a $0.13 \mathrm{~m}$, con el fin de tener árboles homogéneos en sus características físicas. Los árboles que cumplieron con esas características fueron 47. En los 47 árboles estudiados, la altura promedio $( \pm \mathrm{DE})$ fue de $1.6 \pm 0.2 \mathrm{~m}$, la distancia entre árboles de $0.97 \pm 0.26 \mathrm{~m}$; y los diámetros a $0.20 \mathrm{~m}$ y $1.3 \mathrm{~m}$ de altura del suelo de $0.085 \pm 0.021$ y $0.065 \pm 0.018 \mathrm{~m}$, respectivamente. En las dos épocas estudiadas se utilizaron los mismos árboles, los cuales se podaron antes de iniciar el estudio con el fin de obtener uniformidad en la edad de rebrote. La poda se realizó en ambas épocas con pinzas de podar e implicó el corte de todas las ramas. Durante el período de estudio no se aplicó riego, ni fertilizante en suelo. El tipo de suelo es Fluvisol éutrico de textura arcillo-arenoso, localmente conocido como suelo de vega de río (Palma, Cisneros, Moreno, \& Rincon, 2007).

Diámetro del árbol: Con el fin de establecer la participación del diámetro del árbol en las variables respuesta estudiadas, los árboles se clasificaron en dos categorías de acuerdo a su diámetro a los $0.20 \mathrm{~m}$ del suelo: D-9, árboles con un diámetro entre 0.05 y $0.09 \mathrm{~m}(\mathrm{~N}=30$ árboles) y D-13, árboles con un diámetro entre 0.091 y $0.130 \mathrm{~m}(\mathrm{~N}=17)$.

Producción y composición química del follaje: En estudios previos con E. americana, las edades de rebrote de 90 y 120 días produjeron mayor cantidad de MS con relación a 30 y 60 días (Meléndez, 2003). A los 90 días de rebrote, el follaje presenta mayor contenido de PB y menor contenido de carbohidratos estructurales (Hernández-Espinoza et al., 2020a), por lo que, en cada una de las épocas estudiadas, se determinó la producción de follaje árbol ${ }^{-1}$ a los 90 días de rebrote por el método destructivo (corte de todas las ramas y separación del follaje). Una vez cortadas las ramas se procedió a separar el follaje de las mismas, el cual incluyó hojas y peciolos sin incluir tallos tiernos. El follaje y ramas se pesaron en verde (base húmeda) con una báscula de reloj con capacidad para $20 \mathrm{~kg}$ y una sensibilidad de $25 \mathrm{~g}$ (TecnoCor ${ }^{\circledR}$, México, modelo DGN 312.01.2005.2576).

Para determinar la composición química del follaje, en cada época se seleccionaron al azar seis árboles y se obtuvieron muestras de follaje. Las muestras se secaron a $50{ }^{\circ} \mathrm{C}$, se trituraron, pasaron a través de una malla de 1 $\mathrm{mm}$, en molino Wiley, posteriormente a cada una se le determinó por duplicado materia seca (MS), cenizas (Cen), materia orgánica (MO) y PB con métodos de la Asociación de Químicos Agrícolas Oficiales (AOAC, 1999); fracciones de fibra detergente neutro (FDN) y fibra 
detergente ácido (FDA) con las técnicas de Van Soest, Robertson, \& Lewis (1991).

Variables respuesta: En los árboles se midió la producción de follaje árbol ${ }^{-1}$. Se utilizó la información de los kg de follaje cosechado por árbol, MS y PB del follaje para calcular la producción de $\mathrm{MS}$ árbol-1 y $\mathrm{PB}^{-}$ábol $^{-1}$. La relación follaje ramas ${ }^{-1}$ se determinó dividiendo el peso del follaje entre el peso de las ramas, ambas, en base húmeda.

Diseño experimental y análisis estadístico: Todos los análisis se efectuaron con el paquete estadístico SAS v9.3 (SAS, 2002) y se probó la normalidad y homocedasticidad de los datos con las pruebas de Kolmogorov-Smirnov y Levene, respectivamente. Para determinar la influencia de la época del año sobre la composición química del follaje se utilizó un diseño completamente al azar, el factor de estudio fue la época climática (sequía y lluvias). La unidad experimental fue la muestra foliar árbol ${ }^{-1}$. Los datos se analizaron con el procedimiento ANOVA y las medias se compararon con la prueba de Tukey a un $\alpha=0.05$.

Para determinar la influencia de la época climática y diámetro del árbol sobre la producción de follaje se utilizó un diseño experimental de dos factores con medidas repetidas en un factor (Cody \& Smith, 1991). Durante la fase experimental se realizaron mediciones repetidas en los mismos árboles a intervalos de 90 días por lo que se consideró como variable independiente la época climática en la cual se cosechó el follaje (sequía y lluvias). La segunda variable independiente fue el diámetro del árbol (D-9 y D-13) y la interacción entre época y diámetro.

Las variables dependientes producción de MS árbol ${ }^{-1}$ y $\mathrm{PB}$ árbol $^{-1}$ tuvieron normalidad y varianzas homogéneas. Mientras que las variables dependientes producción de follaje árbol ${ }^{-1}$, peso de las ramas cosechadas y relación follaje ramas $^{-1}$ no tuvieron normalidad y varianzas homogéneas, por lo que se transformaron a logaritmo natural (producción de follaje árbol ${ }^{-}$ ${ }^{1}$ ) e inversa (relación follaje ramas $^{-1}$ ). La unidad experimental fue el árbol. Las variables dependientes sin transformar y las transformadas con normalidad y homocedasticidad se analizaron con el procedimiento MIXED. Las medias se compararon con la prueba de " $t$ " con las medias de cuadrados mínimos usando la opción pdiff de SAS y fueron consideradas estadísticamente significativas cuando $\mathrm{P}$ $<0.05$. Las transformaciones utilizadas no permitieron que la variable dependiente peso de las ramas a la cosecha tuviera normalidad y homocedasticidad, por lo que se analizó con el test de la suma de rangos de Wilcoxon para datos no pareados considerando la época de manera independiente (Milton, 2007).

\section{RESULTADOS}

Condiciones climáticas: En la Tabla 1 se describen los valores de temperatura ambiente, humedad relativa, radiación solar y precipitación pluvial durante las dos épocas climáticas.

Composición química del follaje: La época climática afectó el contenido de MS y FDN en el follaje $(\mathrm{P}<0.01)$. El resto de los

TABLA 1

Condiciones climáticas previas a la cosecha de follaje de Erythrina americana a una edad de rebrote de 90 días

\section{TABLE 1}

Weather conditions prior to the harvest of Erythrina americana foliage at a 90-day regrowth age

\begin{tabular}{lcc}
\multicolumn{1}{c}{ Variable } & \multicolumn{2}{c}{ Épocas climáticas } \\
& Sequía & Lluvias \\
Temperatura ambiente $\left({ }^{\circ} \mathrm{C}\right)$ & & \\
$\quad$ Media & 25.8 & 27.9 \\
Mínima & 17.8 & 23.7 \\
Máxima & 32.9 & 32.9 \\
Humedad relativa $(\%)$ & & \\
$\quad$ Media & 81.8 & 85.5 \\
$\quad$ Mínima & 47.0 & 47.0 \\
$\quad$ Máxima & 100.0 & 99.0 \\
Radiación solar $\left(\mathrm{Wm}^{-2}\right)$ & & \\
$\quad$ Media & 197.3 & 209.7 \\
$\quad$ Mínima & 28.5 & 99.2 \\
$\quad$ Máxima & 273.2 & 315.7 \\
Precipitación acumulada $(\mathrm{mm})$ & 268.2 & 583 \\
Número de días & 107 & 100 \\
\hline
\end{tabular}


TABLA 2

Influencia de la época climática sobre la composición química del follaje de Erythrina americana Miller a una edad de rebrote de 90 días

TABLE 2

Influence of the climatic season on the chemistry composition of the Erythrina americana Miller foliage at a 90-day regrowth age

\begin{tabular}{|c|c|c|c|}
\hline \multirow{2}{*}{ Componente } & \multirow{2}{*}{ Número de componentes época ${ }^{-1}$} & \multicolumn{2}{|c|}{ Época climática } \\
\hline & & Sequía & Lluvias \\
\hline Materia seca (\%) & 6 & $32.3^{\mathrm{a}} \pm 1.0$ & $25.9^{b} \pm 0.9$ \\
\hline Materia orgánica $^{1}$ & 6 & $91.5 \pm 0.3$ & $92.0 \pm 0.1$ \\
\hline Cenizas $^{1}$ & 6 & $8.5 \pm 0.3$ & $8.0 \pm 0.1$ \\
\hline Proteína bruta ${ }^{1}$ & 6 & $14.4 \pm 0.3$ & $13.7 \pm 0.3$ \\
\hline Fibra detergente neutro ${ }^{1}$ & 6 & $45.6^{\mathrm{b}} \pm 0.8$ & $50.6^{\mathrm{a}} \pm 0.8$ \\
\hline Fibra detergente ácido ${ }^{1}$ & 6 & $31.6 \pm 0.8$ & $33.2 \pm 0.6$ \\
\hline
\end{tabular}

${ }^{1}$ Porcentaje de la materia seca; a, b Medias con diferente superíndice dentro de la misma hilera difieren $(\mathrm{P}<0.05)$.

TABLA 3

Influencia de la época climática sobre la producción de follaje de Erythrina americana a una edad de rebrote de 90 días

TABLE 3

Influence of the climatic season on the production of Erythrina americana foliage at a 90-day regrowth age

\begin{tabular}{|c|c|c|}
\hline \multirow{2}{*}{ Variable } & \multicolumn{2}{|c|}{ Época climática } \\
\hline & Sequía $(\mathrm{N}=30)$ & Lluvias $(\mathrm{N}=17)$ \\
\hline \multicolumn{3}{|l|}{ Producción de follaje $\left(\mathrm{g}\right.$ árbol ${ }^{-1}$ ) } \\
\hline base húmeda & $794^{b} \pm 68$ & $1049^{a} \pm 68$ \\
\hline base seca & $257 \pm 19$ & $271 \pm 19$ \\
\hline Producción de proteína bruta (g árbol ${ }^{-1}$ ) & $37.0 \pm 3.0$ & $37.0 \pm 3.0$ \\
\hline Relación follaje ramas ${ }^{-1}$ & $2.6^{\mathrm{a}} \pm 0.21$ & $1.8^{\mathrm{b}} \pm 0.21$ \\
\hline
\end{tabular}

$\mathrm{a}, \mathrm{b}$, medias de cuadrados mínimos $\pm \mathrm{EE}$ con letras diferentes dentro de la misma línea indican diferencias $(\mathrm{P}<0.01)$. D-9 = árboles con un diámetro entre 0.05 y $0.09 \mathrm{~m}$; D-13 = árboles con un diámetro entre 0.091 y $0.13 \mathrm{~m} ; \mathrm{N}=$ número de observaciones.

componentes estudiados no fueron influidos por la época $(\mathrm{P}>0.05)$ (Tabla 2).

Influencia de la época y diámetro: $\mathrm{La}$ interacción época $\mathrm{x}$ diámetro del árbol no afectó las variables estudiadas en él árbol ( $\mathrm{P}$ $<0.05)$. La época climática afectó $(\mathrm{P}<0.01)$ la producción de follaje en base húmeda y la relación follaje/ramas. Sin embargo, la producción de follaje en base seca y la producción de proteína bruta foliar no fueron afectadas ( $\mathrm{P}>$ 0.05) (Tabla 3).

Durante la sequía, el diámetro del árbol influyó la producción de ramas $(\mathrm{P}<0.01)$, las medias \pm EE para D-9 y D13 fueron: $233^{\mathrm{b}} \pm$ 33 y $610^{\mathrm{a}} \pm 77 \mathrm{~g}$ árbol $^{-1}$, respectivamente. Una respuesta similar se detectó en lluvias, en D-9 la producción de ramas fue menor con relación a $\mathrm{D}-13(\mathrm{P}<0.01), 438^{\mathrm{b}} \pm 63$ y $996^{\mathrm{a}} \pm 166 \mathrm{~g}$ árbol ${ }^{-1}$, respectivamente.

El diámetro de E. americana influyó (P $<0.01$ ) la producción de follaje (en base húmeda y seca), proteína bruta $\left(\mathrm{g}^{\text {árbol }}{ }^{-1}\right) \mathrm{y}$ la relación follaje/ramas. Los árboles en D-13 produjeron mayor cantidad de MS (g árbol ${ }^{-1}$ ), PB $\left(\mathrm{g}\right.$ árbol $\left.^{-1}\right)$, y tuvieron una menor relación follaje/ramas con respecto a D-9 $(\mathrm{P}<0.01)$. En la Tabla 4 se indican las características físicas 
TABLA 4

Influencia del diámetro del árbol de Erythrina americana sobre la producción de follaje a una edad de rebrote de 90 días

TABLE 4

Influence of the diameter of the Erythrina americana tree on the production of foliage at a 90-day regrowth age

\begin{tabular}{|c|c|c|}
\hline \multirow{2}{*}{ Variable } & \multicolumn{2}{|c|}{ Diámetro del poste $(\mathrm{m})$ sobre el nivel del suelo } \\
\hline & $\mathrm{D}-9(\mathrm{~N}=30)$ & $\mathrm{D}-13(\mathrm{~N}=17)$ \\
\hline Altura (m) & $1.60 \pm 0.02$ & $1.64 \pm 0.03$ \\
\hline \multicolumn{3}{|l|}{ Diámetro del nivel del suelo (m) } \\
\hline 0.20 & $0.07^{\mathrm{b}} \pm 0.002$ & $0.11^{\mathrm{a}} \pm 0.002$ \\
\hline 1.30 & $0.05^{\mathrm{b}} \pm 0.002$ & $0.08^{\mathrm{a}} \pm 0.002$ \\
\hline \multicolumn{3}{|l|}{ Producción de follaje ( $\mathrm{g}$ árbol ${ }^{-1}$ ) } \\
\hline base húmeda & $633^{b} \pm 83$ & $1210^{\mathrm{a}} \pm 98$ \\
\hline base seca & $182^{b} \pm 23$ & $346^{\mathrm{a}} \pm 28$ \\
\hline Producción de proteína bruta (g árbol ${ }^{-1}$ ) & $26.0^{\mathrm{b}} \pm 3.0$ & $48.0^{\mathrm{a}} \pm 4.0$ \\
\hline Relación follaje ramas ${ }^{-1}$ & $2.6^{\mathrm{a}} \pm 0.20$ & $1.8^{\mathrm{b}} \pm 0.23$ \\
\hline
\end{tabular}

a, b, medias de cuadrados mínimos $\pm \mathrm{EE}$ con letras diferentes dentro de la misma línea indican diferencias $(\mathrm{P}<0.01)$. D-9 = árboles con un diámetro entre 0.05 y 0.09 m; D-13 = árboles con un diámetro entre 0.091 y $0.13 \mathrm{~m}$ ); $\mathrm{N}=$ número de observaciones.

y productivas de E. americana considerando el diámetro del árbol.

\section{DISCUSIÓN}

Condiciones climáticas: Durante la sequía, las oscilaciones en la temperatura ambiente, humedad relativa y radiación solar fueron de $15.1^{\circ} \mathrm{C}, 53 \%$ y $244.7 \mathrm{Wm}^{-2}$, respectivamente. Mientras que en la época de lluvias fueron de menor magnitud $9.2{ }^{\circ} \mathrm{C}, 52 \%$ y $216.5 \mathrm{Wm}^{-2}$, respectivamente. La precipitación pluvial acumulada en sequía y lluvia representó el 11.2 y $24.5 \%$ del promedio de precipitación pluvial acumulada anual (2 $386 \mathrm{~mm}$ ) para el municipio de Huimanguillo, Tabasco (Díaz et al., 2006; CONAGUA, 2017). Las fluctuaciones en las condiciones climáticas durante el desarrollo del estudio corresponden a las de transición de las estaciones de nortes a sequía y lluvias en el estado de Tabasco (Moguel \& Molina-Enríquez, 2000; García, 2004; Díaz et al., 2006) y son consideradas como tolerables para el crecimiento y desarrollo de E. americana. Especie que se encuentra distribuida en regiones con clima cálido húmedo, cálido subhúmedo y cálido seco (García-Mateos et al., 2001; CONABIO, 2017).
Composición química del follaje: En términos generales, existe una amplia disponibilidad de especies arbóreas cuyo follaje tiene un uso potencial para utilizar en la alimentación de rumiantes. Sin embargo, este tipo de follajes tiene amplia variación en el contenido de PB (10.9 \% en Mangifera indica L. y 25.8 $\%$ en G. sepium), FDN (25.0 \% en Leucaena leucocephala y $67.6 \%$ en Parmetiera edulis D.C.) y degradabilidad in situ de la MS (33.5 $\%$ en Enterolobium cyclocarpum (Jacq.) Griseb y $82.1 \%$ en G. sepium), lo anterior se atribuye, en parte, a la especie, tipo de suelo, época de cosecha y al desconocimiento de la edad de rebrote del follaje, así como las características de la muestra foliar analizada (con o sin ramas tiernas) (Reyes \& Jiménez, 1998; Pinto-Ruiz et al., 2010; Ramos-Trejo et al., 2016). La amplia variación en los componentes químicos y degradabilidad de la MS de los follajes arbóreos representa una limitante para incluirlos en un programa de complementación alimenticia. En el caso particular de E. americana, los valores de PB en el follaje pueden estar entre 14.5 y $25.6 \%$ (Reyes \& Jiménez, 1998; OlivaHernández et al., 2019), la FDN entre 52.4 y 71.6 \% (Oliva-Hernández et al., 2019; Hernández-Espinoza et al., 2020b) y la degradabilidad 
in vitro de la MS entre 43.5 y $52.6 \%$ (Reyes \& Jiménez, 1998).

El contenido de PB en el follaje fue similar al reportado en follaje de E. americana $\sin$ antecedentes de poda (14.5\%) (Oliva-Hernández et al., 2019) y fue menor al valor indicado con una edad de rebrote similar a la del presente estudio (17.3\%) (Hernández-Espinoza et al., 2020a). Los valores de PB foliar registradas durante las dos épocas estudiadas fueron similares (14.0 \% en Panicum spp.) y mayores a los de gramíneas tropicales (10.6\% en $\mathrm{Bra}$ chiaria spp.) (Enríquez, Meléndez, Bolaños, \& Esqueda, 2011) situación que aumenta el uso potencial del follaje de Erythrina como complemento proteínico para ovinos en pastoreo.

Los promedios generales de FDN y FDA en el follaje fueron inferiores a los indicados en follaje de E. americana, sin antecedentes de poda (entre 52.4 y $60.9 \%$ de FDN; entre 40.1 y $41.9 \%$ de FDA) (Reyes \& Jiménez, 1998; Oliva-Hernández et al., 2019) y con una edad de rebrote de 90 días (52.9 \% de FDN y $37.2 \%$ de FDA) (Hernández-Espinoza et al., 2020a). La época climática afectó el contenido de FDN, una respuesta similar se indica en leguminosas herbáceas (García-Ferrer, Bolaños-Aguilar, Ramos-Juárez, Osorio, \& Lagunes-Espinoza, 2015). Sin embargo, Reyes y Jiménez (1998) indican una ausencia de influencia de la época del año sobre el contenido de carbohidratos estructurales en follaje de E. americana. Otros factores que explican la variación en el contenido de carbohidratos estructurales en el follaje de leguminosas arbóreas son: variación genética dentro de la misma especie, tipo de suelo y clima en el cual crecen los árboles (Wood, Stewart, \& Vargas, 1998). Factores que pudieran explicar las diferencias entre estudios en el contenido de carbohidratos estructurales.

El contenido de Cen y MO se encuentra en los rangos reportados en el follaje de E. americana con $(8.0 \%$ en Cen y $92.0 \%$ en MO a una edad de rebrote de 90 días) (Hernández-Espinoza et al., 2020a) y sin antecedentes de poda (9.4 $\%$ en Cen y $90.6 \%$ en MO) (Oliva-Hernández et al., 2019) y con otras leguminosas arbóreas como Gliricidia sepium (7.9 \% en Cen y 92.1 $\%$ en MO) (Reyes \& Jiménez, 1998).

Es importante considerar que los follajes de especies arbóreas contienen metabolitos secundarios que varían en su naturaleza química y concentración (Pinto et al., 2003; Grande, 2010), este tipo de metabolitos contribuyen al equilibrio en la interacción planta-herbívoro (Ramos, Frutos, Giráldez, \& Mantecón, 1998). Sin embargo, la eficiencia productiva y estado de salud de los rumiantes que consumen follajes con este tipo de metabolitos puede beneficiarse, dañarse o no afectarse. El tipo de respuesta estará condicionada por el número, calidad y concentración de los metabolitos contenidos en el alimento y por la cantidad de alimento que consume el rumiante (Naumann, Tedeschi, Zeller, \& Huntley, 2017; HernándezEspinosa et al., 2020ab). Particularmente, el follaje de Erythrina contiene alcaloides, flavonoides y triterpenoides (Pino et al., 2004), y la edad de rebrote de E. americana afecta la concentración de los polifenoles totales, fenoles no taninos, taninos condensados e hidrolizables. A los 90 días de edad de rebrote del follaje se detecta la menor concentración de taninos condensados (6.0 g kg de MS) con respecto a las edades de rebrote de 60 y 120 días, 9.7 y $14.2 \mathrm{~g} \mathrm{~kg}$ de MS, respectivamente (HernándezEspinosa et al., 2020a).

El consumo de taninos condensados a través del follaje de leguminosas arbóreas puede tener beneficios al animal por sus efectos nematicidas, de protección de las proteínas de la dieta de la degradación ruminal y por reducir los microbios ruminales que producen $\mathrm{CH}_{4}$ (Naumann et al., 2017). Sin embargo, el contenido de taninos condensados en el follaje de leguminosas arbóreas, es afectado por diversos factores (especie, edad de rebrote y condiciones climáticas) lo que puede limitar los beneficios productivos en el animal o en el medio ambiente (Pinto et al., 2003; HernándezEspinosa et al., 2020ab).

La época climática influyó sobre el contenido de MS y FDN foliar. Durante la sequía el contenido de MS fue mayor y la FDN menor con relación a lluvias. El aumento en MS 
durante la sequía coincide con lo indicado en follaje de E. americana procedente de árboles sin antecedentes de poda (Reyes \& Jiménez, 1998). Sin embargo, en el estudio señalado previamente el contenido de la FDN no fue afectada por la época climática. Durante la sequía se presentó una mayor oscilación térmica y la precipitación pluvial represento el $46 \%$ de la detectada en lluvias (Tabla 1), este tipo de condiciones climáticas pudieron favorecer una mayor pérdida de agua foliar por transpiración e incrementar el contenido de MS, lo que explica el aumento de MS (Taiz \& Zeiger, 2002).

Influencia de la época y diámetro: $\mathrm{La}$ producción de follaje en MS y PB foliar no fue afectada por la época climática, la respuesta anterior puede ser explicada por el mayor contenido de MS del follaje durante la sequía y por la menor relación follaje/ramas en lluvias $(\mathrm{P}<0.01)$. Una producción similar de MS y $\mathrm{PB}$ foliar en sequía y lluvias propicia que este recurso alimenticio sea una alternativa para dar sustentabilidad al porcentaje elevado de rebaños ovinos a pequeña escala localizados en la región tropical (Nuncio-Ochoa, Nahed, Díaz, Escobedo, \& Salvatierra, 2001; Pérez et al., 2011; Martínez-Peña, Villagómez-Cortés, $\&$ Mora-Brito, 2018) y en aquellos con posibilidades de utilizar a los árboles multiusos como parte de los cercos (Maldonado et al., 2008; Candelaria-Martínez et al., 2015; MorantesToloza \& Renjifo, 2018).

Por otra parte, el período de sequía en el sitio de estudio tiene una menor duración y mayor precipitación pluvial acumulada con respecto a regiones con clima cálido subhúmedo (Aw) y cálido seco (Bs), en donde la sequía puede ser de hasta seis meses y la precipitación pluvial acumulada en los seis meses de sequía puede estar entre $54.4 \mathrm{~mm}$ (Aw en la vertiente del Pacífico) y $39.4 \mathrm{~mm}$ (en la región de la cuenca de Huajapan, Oaxaca) (García, 2004). Una mayor duración de la sequía y menor precipitación pluvial puede afectar de manera importante el crecimiento y desarrollo de $E$. americana, por lo que es necesario realizar estudios complementarios para determinar su comportamiento productivo en diferentes condiciones climáticas de la región tropical.

En D-13 se detectó mayor producción de MS y PB en el follaje árbol ${ }^{-1}$ en un 190 y 185 $\%$ con respecto a D-9. Un mayor diámetro de tallo favoreció una mayor superficie de los tejidos conductores xilema y floema, el primero conduce el agua, compuestos inorgánicos y orgánicos desde la raíz hacia las hojas y el segundo permite el transporte y reparto de los carbohidratos producidos durante la fotosíntesis, o aquellos movilizadas desde los lugares de almacenamiento, proteínas y reguladores del crecimiento hacia las áreas de crecimiento y almacenaje del árbol (Taiz \& Zeiger, 2002). Una mayor superficie de tejidos conductores de nutrientes en D-13 puede explicar la mayor producción de MS y PB con respecto a D-9.

Con respecto al potencial de producción foliar de E. americana, la presencia de 100 árboles (DAP $0.08 \mathrm{~m}$, distancia entre árboles de $1 \mathrm{~m}$ y una edad de rebrote de 90 días) en $100 \mathrm{~m}$ lineales de cerco vivo permite obtener $34.6 \mathrm{~kg}$ de MS y $4.8 \mathrm{~kg}$ de PB. La MS generada permite cubrir el $15 \%$ del consumo de MS de 312 ovejas con $30 \mathrm{~kg}$ de peso vivo (PV), considerando que el consumo de MS corresponde $24.7 \mathrm{~g} \mathrm{~kg}$ de PV de una oveja que no se encuentra en gestación ni en lactación (Hernández-Espinosa et al., 2020b). Sin embargo, se debe considerar que la poda de los árboles se debe realizar de forma controlada y escalonada con el fin de disponer de follaje todo el año. En un escenario de poda escalonada de los 100 árboles, la poda de 1.1 árboles a intervalos de 90 días permite generar $0.384 \mathrm{~kg}$ de MS foliar día ${ }^{-1}$, cantidad de MS que cubre el $15 \%$ del consumo de MS de 3.5 ovejas con $30 \mathrm{~kg}$ de PV de manera sostenida, en donde la cantidad de follaje ofrecido a cada oveja aporta $15.4 \mathrm{~g}$ de PB, lo que puede representar el $22.3 \%$ de la PB consumida por un ovino alimentado con base en Cynodon plectostachyus (Piñeiro-Vázquez, Oliva-Hernández \& Hinojosa-Cuéllar, 2009).

La relación follaje/ramas se redujo en un $31 \%$ en D-13 con respecto a D-9, lo cual se atribuye a una menor producción de follaje por kilogramo de ramas. En términos generales, la 
relación follaje ramas ${ }^{-1}$ fue mayor a la indicada en árboles de E. americana sin antecedentes de poda (Oliva-Hernández et al., 2019), este tipo de respuesta se atribuye a una menor producción de ramas, debido a que los árboles utilizados se podaron al inicio del estudio (Tabla 2). Entre tanto, en Gliricidia sepium, Leucaena leucocephala (Lam.) de Wit y Guazuma ulmifolia Lam con podas a intervalos entre 75 y 90 días se ha registrado relaciones hoja tallo ${ }^{-1}$ de 3.4, 2.7 y 2.2. respectivamente (Casanova-Lugo, Ramírez-Avilés, \& SolorioSánchez, 2010; Ramos-Trejo et al., 2016), las cuales se encuentran dentro de los valores registrados en el presente estudio. Las diferencias entre estudios pueden atribuirse a la especie, edad de rebrote y a las condiciones de suelo y clima de los sitios en donde se realizaron los estudios mencionados previamente. Una mayor relación follaje ramas ${ }^{-1}$ puede incrementar la eficiencia en la cosecha manual de este tipo de follaje y con ello facilitar la incorporación de este tipo de follaje como complemento alimenticio en rebaños con un número reducido de ovinos (Candelaria-Martínez et al., 2015; Oliva-Hernández et al., 2019; Hernández-Espinoza et al., 2020b).

En conclusión, la época climática afecta el contenido de MS y FDN del follaje de E. americana. Durante la sequía el follaje contiene mayor MS y menor FDN con respecto a lluvias. El diámetro del árbol tuvo mayor influencia que la época en el rendimiento de materia seca y proteína bruta foliar, $\mathrm{kg}$ árbol ${ }^{-1}$. En los árboles con mayor diámetro (D-13) se tiene una mayor producción de follaje y proteína bruta con respecto a los de menor diámetro (D-9). Durante la sequía y lluvias las producciones de MS y PB foliar son similares.

Declaración de ética: los autores declaran que todos están de acuerdo con esta publicación y que han hecho aportes que justifican su autoría; que no hay conflicto de interés de ningún tipo; y que han cumplido con todos los requisitos y procedimientos éticos y legales pertinentes. Todas las fuentes de financiamiento se detallan plena y claramente en la sección de agradecimientos. El respectivo documento legal firmado se encuentra en los archivos de la revista.

\section{AGRADECIMIENTOS}

Los autores agradecen al programa proyectos fiscales del INIFAP por la financiación recibida para la realización del proyecto "Manejo del cerco vivo como proveedor de follaje", clave SIGI: 11391334207 del cual este artículo científico es un entregable.

\section{RESUMEN}

Introducción: Erythrina americana es una leguminosa arbórea que se encuentra como parte del cerco en las fincas ganaderas de la región tropical. Sin embargo, los productores de ovinos no utilizan el follaje de este tipo de leguminosa de forma rutinaria o estratégica como complemento alimenticio debido, en parte, por el desconocimiento de su composición química y rendimiento foliar, así como, por los factores que los afectan. Objetivo: Determinar la influencia de la época climática y diámetro del árbol $E$. americana en la composición química y rendimiento de follaje a una edad de rebrote de 90 días. Método: Se utilizaron 47 árboles en un diseño de dos factores. Los factores fueron época (sequía y lluvias) y diámetro del árbol a 0.20 $\mathrm{m}$ de altura del suelo (D-9 entre 0.05 y $0.09 \mathrm{~m}$ y D-13, entre 0.091 y $0.13 \mathrm{~m}$ ). Las variables respuesta fueron producción de materia seca y proteína bruta foliar $\left(\mathrm{kg}\right.$ árbol $\left.^{-1}\right)$. Resultados: La época no afectó $(\mathrm{P}>0.05)$ la producción de materia seca y proteína bruta. Sin embargo, el diámetro de E. americana si las afectó $(\mathrm{P}<0.01)$. En el diámetro D-13 se detectó la mayor producción de materia seca y proteína bruta foliar con respecto a D-9. Conclusión: El diámetro del árbol tuvo mayor influencia que la época en el rendimiento de materia seca y proteína bruta foliar.

Palabras clave: trópico húmedo; cerco vivo; alimentación de ovinos; leguminosa arbórea.

\section{REFERENCIAS}

AOAC, (1999). Official Methods of Analysis (16th. ed). MD, USA: AOAC International.

Best, D.A., Lara-Lara, P.E., Aguilar-Urquizo, E., CenChuc, F.E., Ku-Vera, J.C., \& Sanginés-García, J.R. (2017). In vivo digestibility and nitrogen balance in sheep diets with foliage of fodder trees in substitution for soybean meal. Agroforest Systems, 91(6), 10791085. DOI: $10.1007 / \mathrm{s} 10457-016-9982-3$ 
Candelaria-Martínez, B., Flota-Bañuelos, C., \& CastilloSánchez, L.E. (2015). Caracterización de los agroecosistemas con producción ovina en el oriente de Yucatán, México. Agronomía Mesoamericana, 26(2), 225-236. DOI: 10.15517/am.v26i2.19278

Casanova-Lugo, F., Ramírez-Avilés, L., \& Solorio-Sánchez, F.J. (2010). Efecto del intervalo de poda sobre la biomasa foliar y radical en árboles forrajeros en monocultivo y asociados. Tropical and Subtropical Agroecosystems, 12(3), 657-665. Recuperado de http://www.revista.ccba.uady.mx/ojs/index.php/TSA/ article/view/190/420

Centurión, H.D., Cázares, C.J.G., Espinosa, M.J., Poot Matu, J.E., \& Mijangos, C.M.A. (2003). Aprovechamiento alimentario de inflorescencias en la región sierra del estado de Tabasco. Polibotánica, 15, 89-97. Recuperado de http://www.redalyc.org/articulo. oa? id $=62101503$

Cody, R.P., \& Smith, J.K. (1991). Applied Statistics and the SAS programming language (3rd ed). New York, USA: Elsevier Science Publishing Co. Inc.

CONABIO. (2017). Colorín, Patol, Zompantle Erythrina americana. Biodiversidad mexicana. Recuperado de http://www.biodiversidad.gob.mx/Difusion/cienciaCiudadana/aurbanos/ficha.php?item=Erythrina $\% 20$ americana

CONAGUA. (2017). Estación Automática Huimanguillo, Tabasco. Servicio Meteorológico Nacional. Recuperado de http://smn1.conagua.gob.mx/emas

Chablé-Pascual, R., Palma-López, D.J., Vázquez-Navarrete, C.J., Ruiz-Rosado, O., Mariaca-Méndez, R., \& Ascensio-Rivera, J.M. (2015). Estructura, diversidad y uso de las especies en huertos familiares de la Chontalpa, Tabasco, México. Ecosistemas y Recursos Agropecuarios, 2(4), 23-39. Recuperado de http:// www.scielo.org.mx/scielo.php?script=sci_arttext\&pi $\mathrm{d}=\mathrm{S} 2007-90282015000100003$.

Díaz, G., Ruíz, J.A., Medina, G., Cano, M.A., \& Serrano, V. (2006). Estadísticas climáticas básicas del estado de Tabasco (Período 1961- 2003) (1ra ed). Veracruz, México: Instituto Nacional de Investigaciones Forestales, Agrícolas y Pecuarias. Recuperado de http://biblioteca.inifap.gob.mx:8080/jspui/bitstream/ handle/123456789/3415/estadisticasclimatologicasbasicasparaelestadodetabasco.pdf?sequence $=1$

Enríquez, J.F., Meléndez, F., Bolaños, E.D., \& Esqueda, V.A. (2011). Producción y manejo de forrajes tropicales (1 ra ed). Veracruz, México: Instituto Nacional de Investigaciones Forestales, Agrícolas y Pecuarias.

Fehling-Fraser, T.C., \& Ceccon, E. (2015). Macropropagation of Erythrina americana in a greenhouse: a potential tool for seasonally dry tropical forest restoration. Revista Chapingo Serie Ciencias Forestales y del Ambiente, 21(1), 5-16. DOI: 10.5154/r. rchscfa.2014.04.016
García-Mateos, R., Soto-Hernández, M., \& Vibrans, H. (2001). Erythrina americana Miller ("Colorín" Fabaceae), a versatile resource from Mexico: A Review. Economic Botany, 55(3), 391-400. DOI: 10.1007/BF02866562

García, E. (2004). Modificación al Sistema de clasificación climática de Köppen (5. ${ }^{\mathrm{a}}$ ed.). D. F., México: Instituto de Geografía, Universidad Nacional Autónoma de México. Recuperado de http://www.igeograf. unam.mx/sigg/utilidades/docs/pdfs/publicaciones/ geo_siglo21/serie_lib/modific_al_sis.pdf

García-Ferrer, L., Bolaños-Aguilar, E.D., Ramos-Juárez, J., Osorio, A.M., \& Lagunes-Espinoza, L. del C. (2015). Rendimiento y valor nutritivo de leguminosas forrajeras en dos épocas del año y cuatro edades de rebrote. Revista Mexicana de Ciencias Pecuarias, 6(4), 453. DOI: 10.22319/rmcp.v6i4.4105

García-Flores, J., González-Espinosa, M., Lindig-Cisneros, R., \& Casas, A. (2019). Traditional medicinal knowledge of tropical trees and its value for restoration of tropical forests. Botanical Sciences, 97(3), 336-354. DOI: 10.17129/botsci.2122

Gómez, M.E., Molina, C.H., Molina, E.J., \& Murgueitio, E. (1990). Producción de biomasa en seis ecotipos de mataratón (Gliricidia sepium). Livestock Research for Rural Development, 2(3). Recuperado de http:// www. lrrd.org/lrrd2/3/gomez.htm

Grande, D., Villanueva, G., Maldonado, N.M., \& Hernández, S. (2013). Las cercas vivas. En M.N. Maldonado (Ed.), Los sistemas silvopastoriles en Tabasco. Una opción para desarrollar una ganadería productiva y amigable con la naturaleza (pp. 23-39). Villahermosa, Tabasco, México: Universidad Juárez Autónoma de Tabasco.

Grande, C.J.D. (2010). Los árboles forrajeros como recurso potencial para el desarrollo de sistemas silvopastoriles en la región de la sierra de Tabasco (Tesis Doctoral). Universidad Autónoma Metropolitana, México, D.F., México.

Hernández-Espinoza, D.F., Lagunes-Espinoza, L. del C., López-Herrera, M.A., Ramos-Juárez, J.A., GonzálezGarduño, R., \& Oliva-Hernández, J. (2020a). Edad de rebrote de Erythrina americana Miller y concentración de compuestos fenólicos en el follaje. Madera y Bosques, 26(1), e2611826. DOI: 10.21829/ myb.2020.2611826

Hernández-Espinoza, D.F., Ramos-Juárez, J.A., GonzálezGarduño, R., Lagunes-Espinoza, L. del C., LópezHerrera, M.A., \& Oliva-Hernández, J. (2020b). Consumo de follaje de Erythrina americana Miller en ovejas Blackbelly x Pelibuey. Revista Mexicana de Ciencias Pecuarias, 11(1), 70-88. DOI: 10.22319/ rmcp.v11i1.5226

INEGI (2017). Anuario estadístico y geográfico de Tabasco. México: INEGI. Recuperado de 
https://www.inegi.org.mx/app/biblioteca/ficha. html?upc $=702825095123$

Llera, M., \& Meléndez, F. (1994). Establecimiento y manejo de cercos vivos en Tabasco. Folleto Técnico 14. Huimanguillo, Tabasco, México: Instituto Nacional de Investigaciones Forestales y Agropecuarias.

Maldonado, M.N., Grande, D.J., Fuentes, E.E., Hernández, S., Pérez-Gil, F., \& Gómez, A. (2008). Los sistemas silvopastoriles de la región tropical húmeda de México: El caso de Tabasco. Zootecnia Tropical, 26(3), 305-308. Recuperado de http://www.scielo.org.ve/ pdf/zt/v26n3/art32.pdf

Martínez-Peña, M., Villagómez-Cortés, J.A., \& MoraBrito, A.H. (2018). Rentabilidad del sistema de producción ovina en el bajo mixe, Oaxaca, México. Agrociencia, 52, 107-122.

Meléndez, F. (2003). Manejo de forrajes tropicales en Tabasco (1 ra ed). Tabasco, México: Instituto Nacional de Investigaciones Forestales, Agrícolas y Pecuarias e Instituto para el Desarrollo de Sistemas de Producción del Trópico Húmedo de Tabasco.

Milton, J.S. (2007). Estadística para biología y ciencias de la salud (3ra ed). Madrid, España: Mc Graw-Hill Interamericana.

Moguel, E.J., \& Molina-Enríquez, J.F. (2000). La precipitación pluvial en Tabasco y Chiapas. Kuxulkab', $5(10), 1-8$.

Morantes-Toloza, J.L., \& Renjifo, L.M. (2018). Cercas vivas en sistemas de producción tropicales: una revisión mundial de los usos y percepciones. Revista de Biología Tropical, 66(2), 739-753. DOI: 10.15517/ rbt.v66i2.33405

Naumann, H.D., Tedeschi, L.O., Zeller, W.E., \& Huntley, N.F. (2017). The role of condensed tannins in ruminant animal production: advances, limitations and future directions. Revista Brasileira de Zootecnia, 46(12), 929-949. DOI: 10.1590/s1806-92902017001200009

Nuncio-Ochoa, G., Nahed, T.J., Díaz, H.B., Escobedo, A.F., \& Salvatierra, I.E.B. (2001). Caracterización de los sistemas de producción ovina en el estado de Tabasco. Agrociencia, 35(4), 469-477. Recuperado de https://www.redalyc.org/pdf/302/30235411.pdf

Oliva-Hernández, J., López-Herrera, M.A., VelázquezJiménez, E., López-Enríquez, G., \& Vélez-Pérez, I.I. (2019). Eficiencia en la cosecha manual de follaje de moté (Erythrina americana Miller). Revista Mexicana de Ciencias Forestales, 10(11), 53-67. DOI: 10.29298/rmcf.v10i51.201

Palma, D.J., Cisneros, J., Moreno, E., \& Rincon, J.A. (2007). Suelos de Tabasco: Su uso y manejo sustentable (1 ra ed.). Villahermosa, Tabasco, México: Colegio de Postgraduados Colegio de Postgraduados, Instituto para el Desarrollo de Sistemas de Producción del Trópico Húmedo de Tabasco, Fundación Produce Tabasco A. C.

Pérez, H.P., Vilaboa, A.J., Chalate, M.H., Candelaria, M.B., Díaz, R.P., \& López, O.S. (2011). Análisis descriptivo de los sistemas de producción con ovinos en el estado de Veracruz, México. Revista Cientifica FCV-LUZ, 11(4), 327-334. Recuperado de http://200.74.222.178/ index.php/cientifica/article/view/15656/15630

Pino, R.S., Prieto, G.S., Pérez, R.E., \& Molina, T.J. (2004). Género Erythrina: Fuente de metabolitos secundarios con actividad biológica. Acta Farmacéutica Bonaerense, 23, 252-258. Recuperado de http://www.latamjpharm.org/trabajos/23/2/ LAJOP_23_2_5_3_5CCQ1E589W.pdf

Pinto, R., Gómez, H., Hernández, A., Medina, F., Martínez, B., Aguilar, V.H., ... Carmona, J. (2003). Preferencia ovina de árboles forrajeros del Centro de Chiapas, México. Pastos y Forrajes, 26(4), 329-334.

Pinto-Ruiz, R., Hernández, D., Gómez, H., Cobos, M.A., Quiroga, R., \& Pezo, D. (2010). Árboles forrajeros de tres regiones ganaderas de Chiapas, México: Usos y características nutricionales. Universidad y Ciencia, 26(1), 19-31. Recuperado de http://www.scielo.org.mx/scielo.php?script=sci 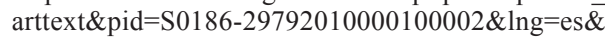 tlng=es

Piñeiro-Vázquez, A.T., Oliva-Hernández, J., \& HinojosaCuéllar, J.A. (2009). Uso de suplementación mineral con monensina sódica en corderas Pelibuey durante el crecimiento postdestete. Archivos de Medicina Veterinaria, 41(1), 35-41. DOI: 10.4067/ S0301-732X2009000100005

Ramos, G., Frutos, P., Giráldez, F.J., \& Mantecón, A.R. (1998). Los compuestos secundarios de las plantas en la nutrición de los herbívoros. Archivos de Zootecnia, 47, 597-620.

Ramos-Trejo, O., Canul-Solis, J.R., \& Ku-Vera, J.C. (2016). Forage yield of Gliricidia sepium as affected by harvest heigh and frequency in Yucatan, Mexico. Revista Bio Ciencias, 4(2), 116-123. DOI: 10.15741/ revbio.04.02.04

Reyes, M.F., \& Jiménez, F.G. (1998). Uso y valor nutritivo de árboles y arbustos con potencial forrajero en la región de la sierra, Tabasco, México. En Reunión Científica Tecnológica Forestal y Agropecuaria. Congreso llevado a cabo en Tabasco, México.

SAS. (2002). Statistical Analysis System, SAS/STAT. En User's Guide (Release 9.3). Cary, North Carolina, USA: SAS Institute.

Sol-Sánchez, A., López-Juárez, S.A., Córdova-Ávalos, V., \& Gallardo-López, F. (2018). Productividad potencial del SAF cacao asociado con árboles forestales. Revista Iberoamericana de Bioeconomía y Cambio Climático, 4(7), 862-877. DOI: 10.5377/ribcc.v4i7.6327 
Sotelo, A., López-García, S., \& Basurto-Peña, F. (2007). Content of nutrient and antinutrient in edible flowers of wild plants in Mexico. Plant Foods for Human Nutrition, 62, 133-138. DOI: 10.1007/ s11130-007-0053-9

Taiz, L., \& Zeiger, E. (2002). Plant physiology (3 ${ }^{\text {rd }}$ ed). Sunderland, USA: Sinauer Associates Inc.

Van Soest, P.J., Robertson, J.D., \& Lewis, B.A. (1991). Methods for dietary fiber, neutral detergent fiber and non-starch polysaccharides in relation to animal nutrition. Journal of Dairy Science, 74, 3583-3597. DOI: $10.3168 /$ jds.S0022-0302(91)78551-2.

Villanueva, G., Martínez, P., \& Van der Wal, H. (2015). Árboles y arbustos en áreas ganaderas de Tabasco: un recurso prometedor. Ecofronteras, 19(54), 14-17.

Wood, C.D., Stewart, J.L., \& Vargas, J.E. (1998). Genetic variation in the nutritive value of Gliricidia sepium: 2. Leaf chemical composition and fermentability by an in vitro gas production technique. Animal Feed Science and Technology, 75(2), 125-143. DOI: 10.1016/S0377-8401(98)00198-9 